\title{
Size-dependent properties of two-dimensional solids
}

\author{
J. A. Zollweg \\ School of Chemical Engineering, Cornell University, Ithaca, New York, 14853 \\ G. V. Chester and P. W. Leung \\ Laboratory of Atomic and Solid State Physics, Cornell University, Ithaca, New York 14853
}

(Received 11 August 1988; revised manuscript received 31 October 1988)

\begin{abstract}
Using Monte Carlo simulations we have found a significant logarithmic size dependence for the density of defects, the shear elastic constant, and the long-range angular order in two-dimensional solids close to their melting points. These size dependencies were present for both hard discs and the repulsive inverse 12th-power potential. Our data suggest that these size dependencies may also be present at higher densities further from the melting density. Simulations very close to melting suggest that the melting densities in the literature are incorrect.
\end{abstract}

\section{INTRODUCTION}

In this paper we present the results from a series of computer simulations on two-dimensional solids. The aim of these simulations was to determine the detailed physical properties of these systems near their melting points. For the purposes of this work we accepted that the melting transition was weakly first order, and we also accepted the best previous estimates of the melting points. Our work was confined to stiff potentials. We have generated very detailed data on the hard-disc (HD) system and the repulsive-inverse 12 th-power $(R-12)$ systems. ${ }^{1}$ We will show that it is possible to map these systems onto each other, thus our results have some degree of universality for stiff potentials. Our basic aim was to examine the behavior of these systems near melting with the goal of shedding some light on the complexities of simulations in this region of the thermodynamic space of the systems. Since the results of direct simulations of the melting-freezing transition tend to point toward a weak first-order transition, ${ }^{1}$ we thought it worthwhile to carry out a fairly exhaustive examination of the solid phase to see whether our data could say anything, even indirectly, about the likely nature of the transition. We believe that a definitive statement about the nature of the transition, based on computer simulations, must await the development of a version of the Monte Carlo (MC) renormalization group method which is applicable to particle systems.

We can easily summarize our results. There are strong size dependences and novel phenomena near melting in the systems we have studied. Our data cast considerable doubt on the validity of simulations of the transition which do not include at least 16000 particles. Our system sizes ranged from 1024 to 16384 particles, and we were able to carry out MC runs of up to two million sweeps for these systems. We found that both the large system size and the long runs are essential if the full range of phenomena were to be revealed. As an essential first step, we determined the pressure of the systems very near melting. Except at one density, all our pressure measurements showed size-independent results - the systems appeared to be in the thermodynamic limit. At one density, about $0.5 \%$ from melting, we found anomalous size dependencies for the HD system. These results may be due to a mislocation of the melting density. ${ }^{2}$ They may also have a more interesting interpretation. However, other properties such as the density of topological defects, the shear elastic constant, the particle displacements, and long-range angular order showed strong size dependencies and anomalous behavior $1 \%$ to $2 \%$ from melting. Some of these results have already been observed by Toxvaerd. ${ }^{3}$ In most instances we have been able to make a more thorough exploration of the configuration space of these systems, and enlarge the data by simulating larger systems.

Our simulations are conducted at constant temperature and density using the standard Metropolis MC algorithm. We used periodic boundary conditions. A word of caution with respect to the simulation of the HD system is necessary. For this system, MC moves are accepted or rejected depending on whether two discs do or do not overlap. Since there are no moves in which the energy increases, no additional random number is generated to determine acceptance or rejection. This essential difference between the HD and other "stiff" potentials means that a very different and predictable sequence of pseudorandom numbers is used in the HD system. Initially, we overlooked the fact and were led astray by long period correlations in our pseudorandom number sequences. We were able to overcome the difficulty in two distinct ways which are described in the Appendix. For the HD system we were able to make runs with two million sweeps. The code for the $R-12$ system was considerably slower. For this system we made runs of 300000 sweeps. For both systems the codes were very carefully optimized for the FPS M64 processors which are part of the Cornell National Supercomputer Facility. For both systems we computed the pressure, density of topological defects, the mean-square displacements of the particles, and the local and long-range angular order. For the $R-12$ system we also computed the shear elastic constant. 
In Sec. II we present our data on the pressure. Section III is devoted to a calibration of the $R-12$ and HD system based on the pair-correlation functions. In Sec. IV we present our data on the density of defects. Sections V and VI deal with the shear elastic constant and the particle displacements, respectively. The data on local and long-range angular order is described in Sec. VII. We present an overview of our data and suggest directions for future work in Sec. VIII.

\section{THE PRESSURE}

The focus of this paper is on the structural properties of two-dimensional solids. As a preliminary step, we present our data on the equations of state of the HD and $R-12$ systems. Our aim was to determine whether there was any appreciable size dependence in the equation of state and whether these systems were stable over very long MC runs. For both systems we assumed that the "conventional" melting densities were correct. For the HD system we assumed that the melting density was given by $\rho \sigma^{2}=\rho^{*}=0.912 .^{2}$ For the $R-12$ system, Weeks ${ }^{1}$ has provided two estimates, $\rho^{*}=1.008$ and $\rho^{*}=1.011$. We took $\rho^{*}=1.01$ for our reference melting density.

Table I shows our results for the HD system at two densities, $\rho^{*}=0.923$ and $\rho^{*}=0.942$, and for system sizes of 1024,4096 , and 16384 particles. The data show that there is a small size dependence of about $0.1 \%$. Figure 1 shows the control chart for the 16384-particle system at a density of $\rho^{*}=0.923$. In this system there is a very small drift of about $0.1 \%$ in the pressure over this very long run. For the two smaller systems there is no drift. The same conclusions are valid for the $R-12$ system. Table II shows the pressure in this system at $\rho^{*}=1.02$, 1.04, and 1.06. Again, there is almost no size dependence. Runs were carried out to only 300000 sweeps. In these shorter runs there is no pressure drift. We will show later that $\rho^{*}=1.04$ is the density in the $R-12$ system, which is comparable to $\rho^{*}=0.923$ in the HD system.

For the HD system we have done several long runs at a density $\rho^{*}=0.917$. This density is $0.5 \%$ from melting and is thus very much closer to melting than those states discussed above. We now find a significant size dependence. The pressure for 1024 particles is 7.875 in reduced units. For a 4096-particle system the pressure over two

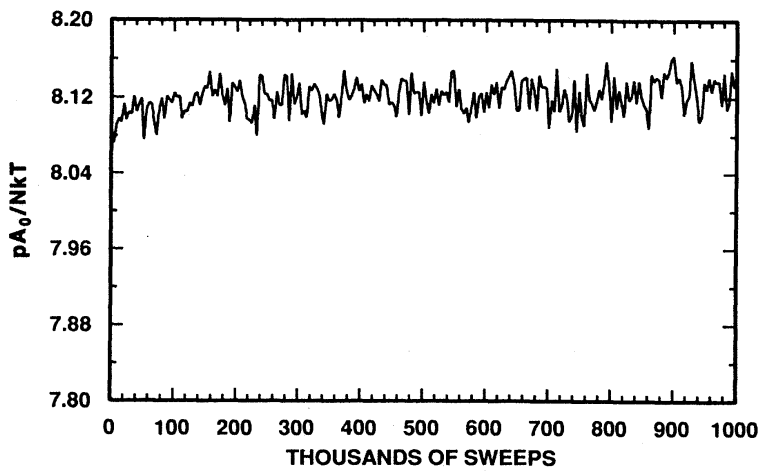

FIG. 1. The control chart for the pressure of the HD system with 16384 particles and one million sweeps; $\rho^{*}=0.923 . A_{0}$ is the area at close packing.

million sweeps has an average of $8.005 \pm 0.027$. This difference amounts to $2 \%$. For the 4096-particle system the pressure changes throughout a two million sweep run. The change is about $0.5 \%$ between the first half and the second half of the run. Table III shows the details of this long run. The control chart is shown in Fig. 2. The pressure has a slow oscillation with an amplitude of about $3 \%$. From the control chart and the data in Table III we are forced to conclude that we have not been able to determine the equilibrium value of the pressure in this system. We will see later that the evolution of the pressure is closely linked to the degree of disorder in the system as measured by the density of topological defects. We have also studied the pressure at this density in the 16 384-particle system. The data are in Table III. The mean value, ignoring the first 200000 sweeps, is $7.972 \pm 0.007$. This value is thus within the error bar of the value of the pressure in the 4096-particle system, $8.005 \pm 0.027$. The pressure again tends to drift upward. The value over the first portion of the run is 7.955 , over the second portion 7.985 , a difference of about $0.5 \%$. We again conclude that we have not found the equilibrium value of the pressure; the system is still evolving. We can summarize our results as follows. The pressure in the 1024-particle system is clearly low. We will see that little diffusion is taking place, and the system is probably clamped by the boundary conditions. The pressures in

TABLE I. The pressure in the HD system in units of $N k T / A_{0}$ and for the densities $\rho^{*}=0.923$ and $\rho^{*}=0.942 . N_{P}$ is the total number of sweeps in the run, $N_{B}$ is the number of sweeps in a block used to determine the uncertainty in the data, and $N_{R}$ is the number of sweeps which were not included in the averaging process. $N$ is the number of particles in the system.

\begin{tabular}{lrcccc}
\hline \hline$\rho^{*}$ & $N$ & $10^{-3} N_{P}$ & $10^{-3} N_{B}$ & $10^{-3} N_{R}$ & $P A_{0} / N k T$ \\
\hline 0.923 & 256 & 1000 & 1000 & 0 & $8.117 \pm 0.008$ \\
& 1024 & 1000 & 1000 & 0 & $8.113 \pm 0.004$ \\
& 4096 & 1000 & 200 & 200 & $8.111 \pm 0.005$ \\
0.942 & 16384 & 1000 & 200 & 200 & $8.120 \pm 0.005$ \\
& 1024 & 1000 & 1000 & 0 & $8.959 \pm 0.004$ \\
& 4096 & 1000 & 200 & 200 & $8.958 \pm 0.002$ \\
& 16384 & 1000 & 200 & 200 & $8.963 \pm 0.003$ \\
\hline \hline
\end{tabular}


TABLE II. The pressure in the $R-12$ system at the densities $\rho^{*}=1.02,1.04$, and 1.06 in units of $k T / \sigma^{2} . N, N_{P}, N_{B}$, and $N_{R}$ are defined in Table I.

\begin{tabular}{lrcccc}
\hline \hline$\rho^{*}$ & $N$ & $10^{-3} N_{P}$ & $10^{-3} N_{B}$ & $10^{-3} N_{R}$ & $P \sigma^{2} / k T$ \\
\hline 1.02 & 256 & 300 & 50 & 50 & $15.193 \pm 0.014$ \\
& 1024 & 300 & 50 & 50 & $15.213 \pm 0.008$ \\
& 4096 & 300 & 50 & 0 & $15.232 \pm 0.009$ \\
1.04 & 16384 & 300 & 50 & 50 & $15.245 \pm 0.009$ \\
& 1024 & 300 & 50 & 50 & $16.625 \pm 0.010$ \\
1.06 & 4096 & 300 & 50 & 50 & $16.648 \pm 0.008$ \\
& 256 & 300 & 50 & 50 & $18.226 \pm 0.012$ \\
& 1024 & 300 & 50 & 50 & $18.212 \pm 0.011$ \\
& 4096 & 300 & 50 & 50 & $18.235 \pm 0.003$ \\
& 16384 & 200 & & 0 & $18.234 \pm 0.006$ \\
\hline \hline
\end{tabular}

the larger systems have overlapping error bars. Both systems are still evolving, and we have not been able, to at least $0.5 \%$, to determine the equilibrium pressure. We also note the relatively large fluctuations in the pressure in the 4096-particle system. Instead of being twice as large as those in the 16384-particle system, they are four times as large. This observation strongly supports our contention that these systems are not in thermodynamic equilibrium. Clearly, there must be another mechanism operating to account for this extra factor of 2 . The larger system seems to be evolving more smoothly. This may be because the boundary conditions are less important and thus the interior of the system is less constrained. It is interesting to note that this smoother evolution leads to less diffusion than in the system with 4096 particles.

\section{THE PAIR DISTRIBUTION FUNCTION- CALIBRATING THE SYSTEMS}

The two systems which we have studied have different interparticle potentials. However, since the inverse 12th-power potential $(R-12)$ is similar to the hard-disc (HD) potential, it is plausible that one might be able to find a fairly precise way of comparing the data for the two systems. We chose to calibrate the two systems by trying to match the pair distribution functions. For a system of 1024 particles we can obtain an accurate pair

TABLE III. The pressure in the HD system in units of $N k T / A_{0}$ at $\rho^{*}=0.917$ and for two system sizes. The pressure is averaged over the number of sweeps shown in the first column.

\begin{tabular}{ccc}
\hline \hline $10^{3}$ Sweeps & $N=4096$ & $N=16384$ \\
\hline $0-200$ & 7.910 & 7.907 \\
$200-400$ & 7.952 & 7.933 \\
$200-400$ & 7.952 & 7.933 \\
$400-600$ & 7.950 & 7.956 \\
$600-800$ & 8.002 & 7.959 \\
$800-1000$ & 8.033 & 7.977 \\
$1000-1200$ & 7.984 & 7.985 \\
$1200-1400$ & 7.958 & 7.980 \\
$1400-1600$ & 8.070 & 7.974 \\
$1600-1800$ & 8.062 & 7.974 \\
$1800-2000$ & 8.048 & 8.017 \\
\hline \hline
\end{tabular}

correlation function out to 15 neighbors. This function displays the basic character of the positional order. In Fig. 3 we show the pair distribution function for the HD system at $\rho^{*}=0.923$. Our basic idea was to accurately match a pair distribution function in the HD system with a pair distribution function in the $R-12$ system. We might then expect that this would lead to a calibration of the densities of the two systems. Since the potentials are different, we do not expect to match the functions at short distances; however, we may expect to match them at intermediate and large distances. We first computed the difference in $g(r)$ for the HD system at two neighboring densities, $\rho^{*}=0.923$ and $\rho^{*}=0.942$. This difference $\Delta g$ tells us how much $g(r)$ changes in the reference HD system when we change the density by about $2 \%$. We refer to this difference, which we will denote by $\widetilde{\Delta} g$, as the reference $\Delta g$. Our task is to match the distribution functions in the two systems so that the difference $\Delta g$ between the two systems is very small compared with $\widetilde{\Delta} g$. We computed the difference between $g(r)$ in the HD system at $\rho^{*}=0.923$ and $g(r)$ in the $R-12$ system at several different densities. The $R-12$ system at a reduced density of $\rho^{*}=1.04$ yields a $\Delta g$ which is very small compared with $\widetilde{\Delta} g$. Figure 4 shows our results. Clearly, we have not matched very well at short distances, but at intermediate and large distances $\Delta g$ is very small compared with $\widetilde{\Delta} g$. We therefore conclude that it is reasonable to compare

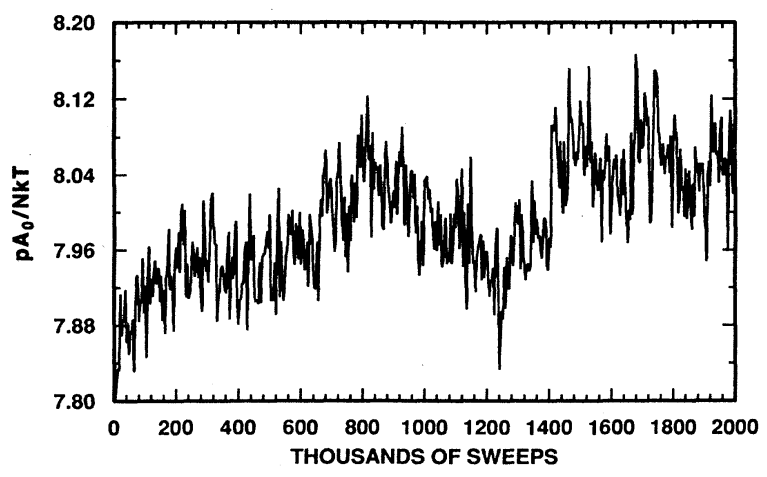

FIG. 2. The control chart for the pressure of the HD system with 4096 particles and two million sweeps; $\rho^{*}=0.917$. 


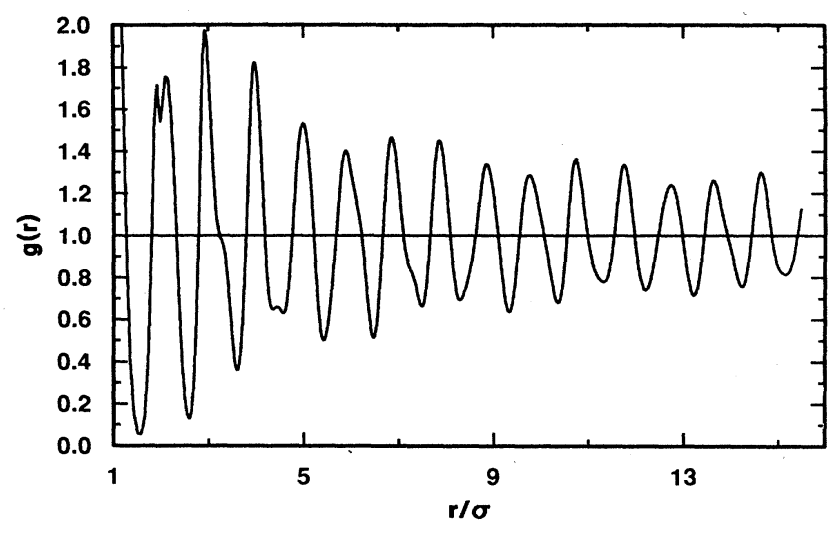

FIG. 3. The pair distribution function, $g(r)$, for the HD system; $\rho^{*}=0.923$.

the physical properties of the two systems at these two densities. We will see that they are indeed very similar. However, we must immediately point out that at a density of $\rho^{*}=0.923$ the HD system is $1 \%$ from the melting density; whereas at the density of $\rho^{*}=1.04$ the $R-12$ system is $3 \%$ from melting. Our successful calibration is thus telling us that at these rather different relative densities the pair functions show almost the same intermediate and long-range order.

Before proceeding further we must clarify one point concerning the $R-12$ system. The calibration we have carried out was done with data generated on the $T^{*}=1$ isotherm. One might wonder whether such calibrations can be carried out on other isotherms, or for that matter, whether we have been especially fortunate in choosing a particular isotherm. It is easy to see that the calibration we have carried out has a universal character. This is because the thermodynamic states of the $R-12$ system can be described by a single variable $\Gamma$. This is the wellknown scaling theorem for inverse power potentials. ${ }^{4}$ For the $R-12$ system a quantity $\Gamma$ can be defined by the equation

$$
\Gamma=\left(\pi \rho^{*}\right)^{6} / T^{*}
$$

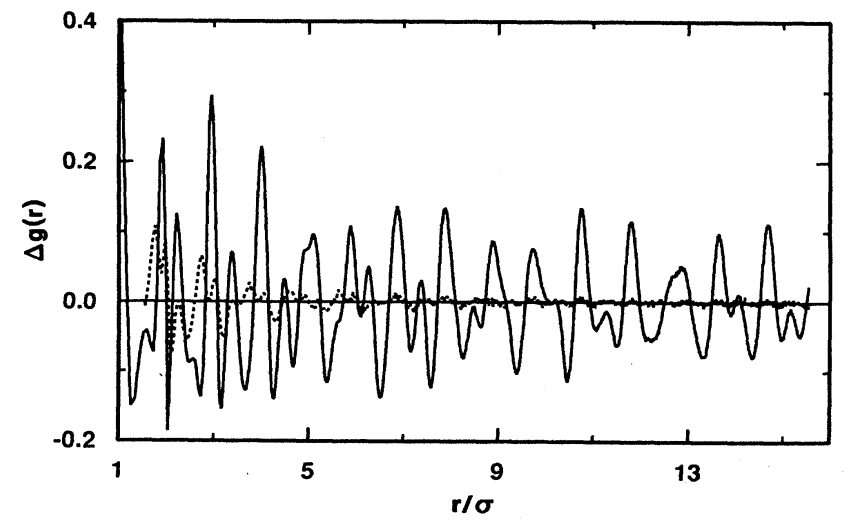

FIG. 4. The reference difference $\Delta \widetilde{g}$ (solid curve) for the HD system and $\Delta g$ (dotted curve) computed from $g(r)$ for the HD system at $\rho^{*}=0.923$ and $g(r)$ for the $R-12$ system at $\rho^{*}=1.04$.
The scaling theorem then states that all physical properties, when measured in reduced units, are a function of $\Gamma$ alone. Thus, the $T^{*}$ and $\rho^{*}$ variables combine into a single variable $\Gamma$. For example, the melting line of the system is given by the equation

$$
\Gamma=\text { const . }
$$

This defines the melting curve in the $\rho^{*}-T^{*}$ plane. If we fix $T^{*}$ by choosing an isotherm and then choose a $\rho^{*}$, we have then determined $\Gamma$. Thus, the calibration we have carried out at $T^{*}=1$ and $\rho^{*}=1.04$ tells us that we have calibrated successfully at some value of $\Gamma(\Gamma=1216.5)$. But since the physical properties, including $g(r)$, depend only on $\Gamma$, we are assured that the calibration will be equally accurate on any isotherm we choose, provided we select the correct reduced density. Moreover, since the value of $\Gamma$ at which the original matching was carried out is known, we can for any different isotherm immediately compute the $\rho^{*}$ at which the calibration will be exactly the same.

We have been able to carry out this matching at two other density pairs. We find that $\rho^{*}=0.917$ (HD) matches very well with $\rho^{*}=1.02(R-12)$ and that $\rho^{*}=0.942$ (HD) matches well with $\rho^{*}=1.08(R-12)$. Clearly, the density scales in the two systems are related in a highly nonlinear fashion. We will see that this method of matching or calibrating the two systems does allow us to make an almost quantitative comparison of their physical properties.

\section{TOPOLOGICAL DEFECTS}

We have computed the density of topological defects for both the HD and for the $R-12$ systems. The defect densities were determined for systems with $N=256$, 1024, 4096, and 16384 particles. The techniques for counting the defects in a two-dimensional system are now well established. ${ }^{5}$ A code, which constructs the Voronoi polygons, was tested against other codes, and in all our runs yielded results consistent with the neutrality requirement. ${ }^{6}$ Tables IV and $\mathrm{V}$ give the details of our Monte Carlo runs. For the HD code we were able to make runs of at least one million sweeps for each system. The code for the $R-12$ system is considerably slower, and we limited ourselves to runs of 300000 sweeps. In each case we were able, by block averaging, to obtain an accurate estimate of the error in our computations. The density of defects was defined as the ratio of $\left(2 N_{4}+N_{5}\right) / N$. Here, $N_{4}$ and $N_{5}$ are the number of four-sided and five-sided polygons respectively. $N$ is the total number of particles in the system. Since the system must be neutral with respect to defects, $2 N_{4}+N_{5}$ must equal $\sum_{i>6} N_{i}(i-6)$. We have not observed any polygons with three sides. For the $R-12$ system we held the reduced temperature constant at $T^{*}=1$, and on this isotherm we worked at three densities, $\rho^{*}=1.02,1.04$, and 1.06. These are $1 \%, 3 \%$, and $5 \%$, respectively, above the melting density. The first two of these densities calibrate accurately with densities in the HD system of $\rho^{*}=0.917$ and $\rho^{*}=0.923$, respectively.

We will focus our attention on (i) a comparison of the 
TABLE IV. The density of topological defects in the HD system at $\rho^{*}=0.923$ and $\rho^{*}=0.942$. The symbols $N, N_{P}, N_{B}$, and $N_{R}$ have been defined in Table I.

\begin{tabular}{rrcccc}
\hline \hline$\rho^{*}$ & $N$ & $10^{-3} N_{P}$ & $10^{-3} N_{B}$ & $10^{-3} N_{R}$ & $10^{3}$ defect density \\
\hline 0.923 & 256 & 1000 & 40 & 0 & $6.76 \pm 0.59$ \\
& 1024 & 1000 & 40 & 0 & $6.54 \pm 0.37$ \\
& 4096 & 1000 & 16 & 200 & $6.73 \pm 0.12$ \\
0.942 & 16384 & 1000 & 16 & 200 & $7.34 \pm 0.13$ \\
& 1024 & 1000 & 40 & 0 & $2.21 \pm 0.26$ \\
& 4096 & 1000 & 16 & 200 & $1.99 \pm 0.06$ \\
& 16384 & 1000 & 16 & 200 & $2.16 \pm 0.01$ \\
\hline \hline
\end{tabular}

density of defects in the two systems at fixed $N=1024$, (ii) the variation of the density of defects with the particle density again at $N=1024$, and (iii) the size dependence of the defect density.

\section{A. The density of defects}

In Fig. 5 we plot the density of defects for the two systems against a nonlinear particle-density axis. All the data are for systems of 1024 particles. The purpose of this plot is to show that when the particle densities in the two systems are matched in the way we described in Sec. III, then the defect densities are in semiquantitative agreement. This is revealed by the two curves following parallel paths which are not very far apart. It is important to notice that there are two matched density scales for the $x$ axis in the plot. From this diagram we can conclude that our matching procedure works well. In other words, to achieve this calibration we have shifted the relative densities of the two systems. For example, the HD system at $\rho^{*}=0.917$ matches the $R-12$ system at $\rho^{*}=1.02$.

\section{B. Density of defects as a function of particle density}

In Fig. 6 we plot the density of defects as a function of particle density. This figure is of course another version of Fig. 5. However, since we are no longer comparing the two systems, we are free to choose a suitable density scale to reveal the actual dependence on particle density. Figure 6 shows that in both systems the defect density follows an exponential law very accurately. There is thus a very rapid variation of defect density with particle density. The coefficient of the exponent differs by a factor of 2 between the HD and $R-12$ systems. It is nevertheless remarkable that both defect densities decrease with in- creasing density in an exponential manner. This is the first time that the defect density has been accurately determined as a function of particle density. A very remarkable property of the two systems is revealed by this plot. When both curves are extrapolated to the melting density, they intersect. Provided our estimates of the melting densities are correct, we can conclude that at melting both systems have the same density of defects. However, we must recall that all the data shown in Fig. 6 are for a system of 1024 particles. As we have already pointed out, for larger systems, near melting, we cannot establish an equilibrium density of defects. More extended simulation studies will be carried out to investigate these new equilibrium situations.

\section{Size dependence}

The size dependence of the density of defects in the Lennard-Jones (LJ) system has been studied by Toxvaerd. ${ }^{3}$ Our data are in qualitative agreement with his, although we have studied larger systems. Our data for both the HD and $R-12$ systems are shown in Fig. 7. Here we have plotted the density of defects versus the logarithm of the size of the system. At the high densities of $\rho^{*}=1.06$ for the $R-12$ system and $\rho^{*}=0.942$ for the HD system there is no significant size dependence. At the lower densities, about $1 \%$ from melting, both systems show a significant size dependence. For the $R-12$ system at $\rho^{*}=1.02$ there is a very clear $\log N$ dependence. The HD system shows an approximate $\log N$ behavior. We need to study a still larger system to confirm this $\log N$ behavior. This size dependence is similar to that observed by Toxvaerd. Clearly, it must be taken into account in any theory or simulation study of the melting phenomenon. One plausible explanation is that it is relat-

TABLE V. The density of topological defects in the $R-12$ system at $\rho^{*}=1.02$ and $\rho^{*}=1.06$. The symbols $N, N_{P}, N_{R}$, and $N_{B}$ have been defined in Table I.

\begin{tabular}{lrcccc}
\hline$\rho^{*}$ & $N$ & $10^{-3} N_{P}$ & $10^{-3} N_{B}$ & $10^{-3} N_{R}$ & $10^{3}$ defect density \\
\hline 1.02 & 1024 & 300 & 50 & 50 & $9.64 \pm 0.21$ \\
& 4096 & 300 & 50 & 0 & $10.92 \pm 0.35$ \\
1.06 & 16384 & 300 & 50 & 100 & $11.88 \pm 0.29$ \\
& 1024 & 300 & 50 & 0 & $2.60 \pm 0.07$ \\
& 4096 & 300 & 50 & 0 & $2.75 \pm 0.02$ \\
& 16384 & 200 & 50 & 0 & $2.79 \pm 0.04$ \\
\hline \hline
\end{tabular}




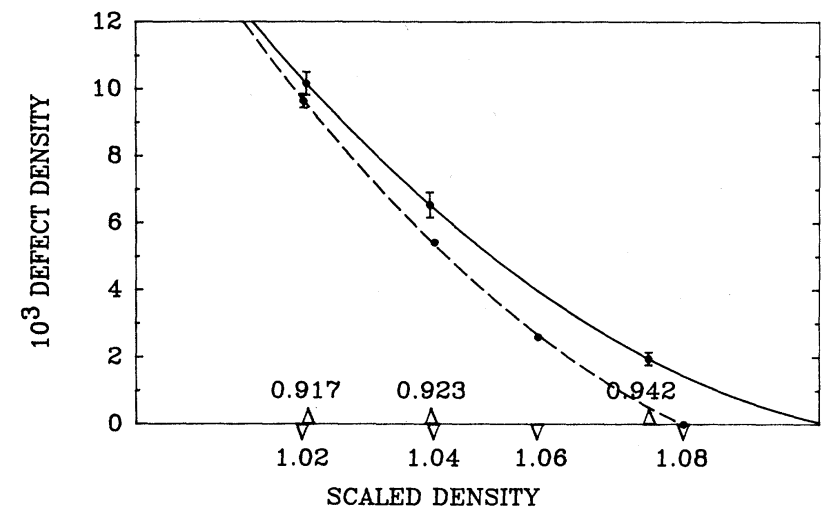

FIG. 5. The defect density as a function of particle density. All the data are for systems with 1024 particles. The density axis shows the calibrated densities for the two systems. The data for the HD system are shown by the continuous curve, that for the $R-12$ system by the dashed curve.

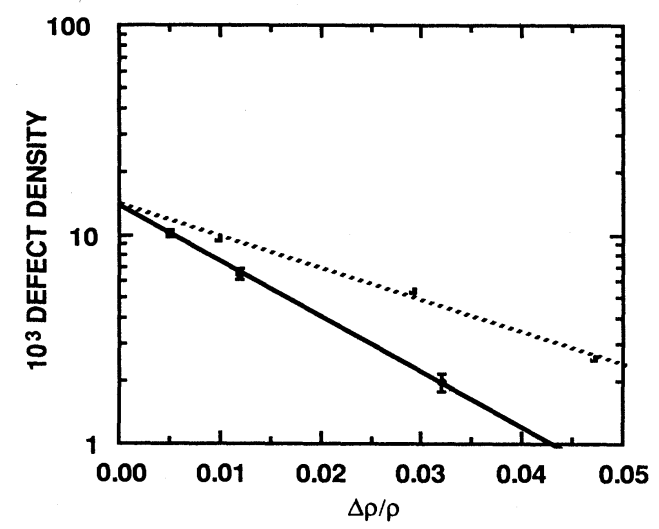

FIG. 6. The defect density for the HD and $R-12$ system as a function of particle density, $\Delta \rho=\rho-\rho_{m}$, where $\rho_{m}$ is the melting density. All the data are for systems of 1024 particles. The data for the HD system are shown by the continuous line, those for the $R-12$ system by the dashed line.

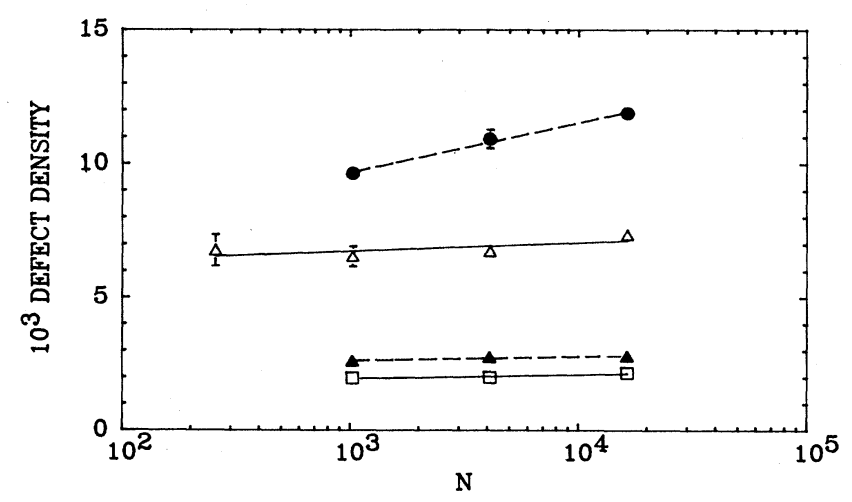

FIG. 7. The size dependence of the density of defects. Shown are the HD system at $\rho^{*}=0.923(\Delta)$, at $0.942(\square)$, and the $R-12$ system at $\rho^{*}=1.02(\bullet)$ and at $1.06(\Delta)$. ed to defects unbinding very near melting. However, it may also be related to the logarithmic increase in the - mean-square amplitude of the lattice displacements (see Sec. VI). We have also computed the density of defects in the HD system at $\rho^{*}=0.917$, about $0.5 \%$ from melting. The data for the 1024-particle system have already been displayed in Fig. 5. During a one million sweep run this system did not show any appreciable drift in the density of defects. However, the density of defects shows appreciable drift for the 4096-particle system. In Fig. 8 we show the control chart for a two million sweep run. The density of defects shows fairly large oscillations over 500000 passes, and it has increased by about $50 \%$ over the course of the run. The system is clearly becoming disordered. It is not possible to quote a meaningful number for the mean number of defects in this size system when it is so close to melting. Figure 9 shows the control chart for the defect density in the 16384-particle system. Again, there is a clear drift upward, of about $50 \%$ in the density during the run, and again we cannot quote an equilibrium value for the defect density. A comparison of Figs. 8 and 9 shows that the fluctuations in the defect density are much less in the 16384-particle system when compared with the 4096-particle system. However, the reduction is much larger than expected from the theory of thermodynamic fluctuations, a factor of 4 instead of 2 . We saw exactly the same phenomenon in the pressure fluctuations at this density. We again remark that the behavior of the fluctuations in defect density as a function of system size tells us that these systems have not reached thermodynamic equilibrium.

It is interesting to compare the control chart shown in Fig. 8 with the corresponding control chart for the pressure Fig. 2. In both the 4096- and 16 384-particle systems there is a remarkably close correlation in the behavior of the pressure and defect density. This correlation tells us that the variation of the pressure is closely linked to the disorder in the system, as measured by the defect density. We believe that as the disorder increases, we have more close encounters of the hard discs which leads to an increase in the pressure. Conversely, when the disorder decreases, there are fewer close encounters, and the pressure drops. This remarkably simple explanation rests, of

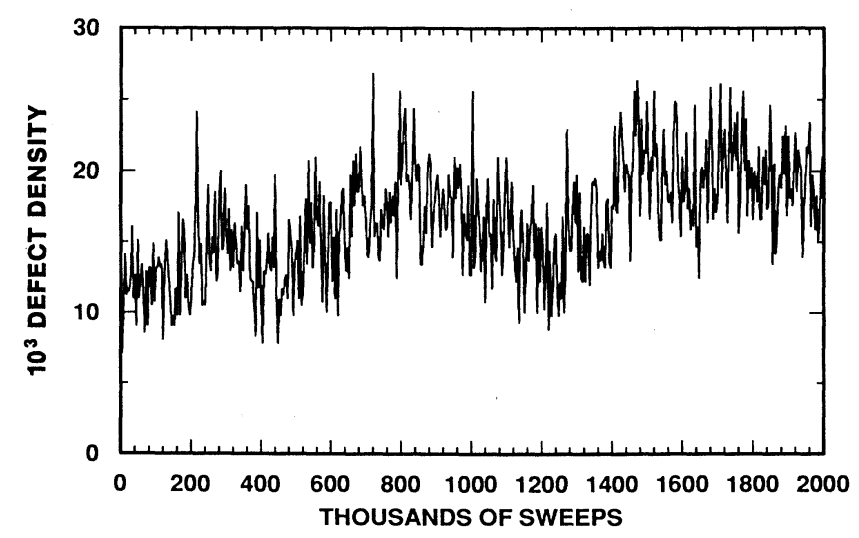

FIG. 8. The control chart for the density of defects for 4096 particles and two million sweeps; $\rho^{*}=0.917$. 


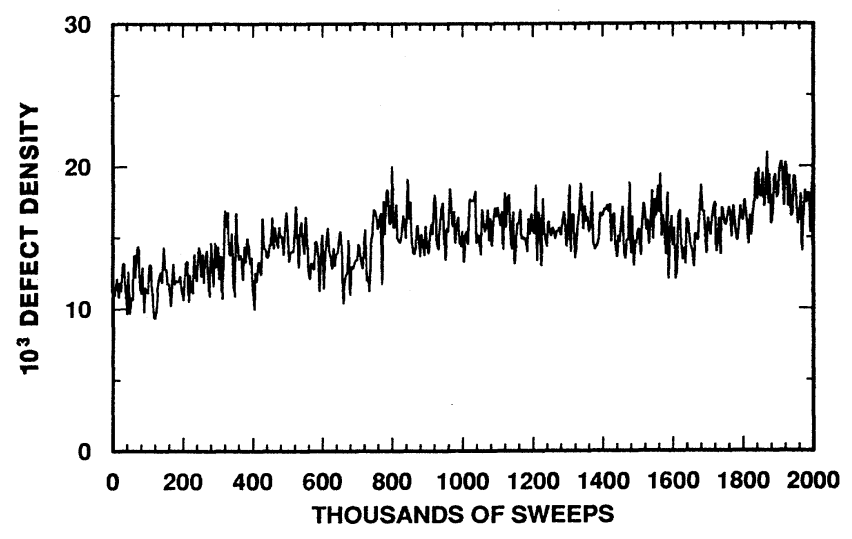

FIG. 9. The control chart for the density of defects for 16384 particles and two million sweeps; $\rho^{*}=0.917$.

course, on the fact that the pressure in a hard-disc system is proportional to the pair distribution function at contact. If we can understand the evolution of the disorder in the system, we will be able to explain the evolution of the pressure.

We end this section with the remark that the HD system at this density may in fact be slowly melting. We base this observation on the fact that during these long runs the disorder is slowly increasing. If the system is indeed slowly melting, then the conventional melting density of 0.912 is incorrect.

\section{THE SHEAR ELASTIC CONSTANT}

We have computed the shear elastic constant for the $R-12$ system at two densities and for systems with $N=256,1024,4096$, and 16384 particles. The method we used was the same as that used by Weeks, ${ }^{1}$ namely small strains were applied to the system and the resulting stress was measured in a long MC run. In this way a linear relation between stress and strain was established. The coefficient of this relation is the shear constant. This method was found to be much more accurate then a direct computation based on the Squire formulas. ${ }^{7}$ This latter method produces data with very large fluctuations. Figure 10 shows the data at $\rho^{*}=1.02$ and $\rho^{*}=1.06$. These densities are $1 \%$ and $5 \%$ above melting. At $5 \%$ from melting there is no size dependence. However, at $1 \%$ from melting there is a very clear logarithmic size dependence. The shear modulus decreases logarithmically with system size. This phenomenon was also observed by Toxvaerd, ${ }^{3}$ but was based on more limited data. We think it plausible that the size dependence is directly related to the similar size dependence we found for the density of topological defects (see Sec. IV). We have not carried out a corresponding study for the HD system, but plan to do so in the near future.

We have also estimated the size dependence of the bulk modulus or isothermal compressibility. We need to measure $(\partial p / \partial \rho)_{T}$; we estimated this quantity by computing the pressure $p$ at two closely spaced densities. Our data showed only a small size dependence for the bulk modulus. The compressibility is related to the Lamé con-

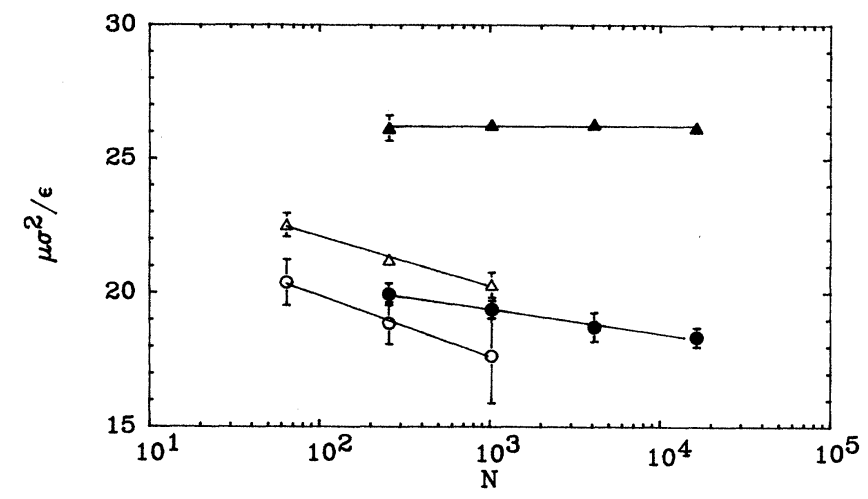

FIG. 10. The size dependence of the shear elastic constant $\mu$. The data are shown as follows: $(\Delta) R-12$ at $\rho^{*}=1.06$; () $R-12$ at $\rho^{*}=1.02 ;(\Delta) \mathrm{LJ}$ at $\rho^{*}=0.937 ;(\bigcirc) \mathrm{LJ}$ at $\rho^{*}=0.929$. The LJ data are taken from Ref. 3.

stants $\lambda$ and $\mu$ by the relation

$$
\rho(\partial p / \partial \rho)_{T}=\lambda+\mu \text {. }
$$

This relation allows us to directly compute the size dependence of $\lambda+\mu$. Table VI shows this dependence along with that for $\mu$.

In the Kosterlitz-Thouless-Halperin-Nelson-Young (Ref. 8) theory of melting, the quantity $K$. defined by the equation

$$
K=\frac{8}{\sqrt{3}} \frac{\beta}{\rho} \frac{\mu}{1+\frac{\mu}{\mu+\lambda}}
$$

plays an important role. Melting is predicted to occur when $K$ reaches the value of $16 \pi .^{8}$ This is a theoretical result valid in the large $N$ or thermodynamic limit. Our data on $\lambda+\mu$ and $\mu$ lead to a fairly strong size dependence for $K$ which is also shown in Table VI. This size dependence carries the clear warning that computer simulations of the melting phenomena may well be misleading unless large systems are simulated and great care is taken to examine size dependence.

It is worthwhile pointing out that the decrease in shear modulus which we have observed may have a plausible explanation. In a two-dimensional lattice a free dislocation must span the entire system. This implies that when the system is stressed, it can readily shear along the dislocation and will then appear to be soft. We must therefore expect that very near melting, where we observe free dislocations, we must expect a "softening" of the system. As the density of these defects increases, we must expect that the system will become softer and softer. We believe

TABLE VI. The elastic constants in the $R-12$ system at $\rho^{*}=1.02$. The quantities $\mu, \lambda$, and $K$ are defined in the text, $\varepsilon$ is the strength of the $R-12$ potential, and $\sigma$ is its length scale.

\begin{tabular}{rccc}
\hline \multicolumn{1}{c}{$N$} & $\mu \sigma^{2} / \varepsilon$ & $(\lambda+\mu) \sigma^{2} / \varepsilon$ & $K$ \\
\hline 256 & $19.94 \pm 0.41$ & 72.2 & 70.8 \\
1024 & $19.37 \pm 0.33$ & 70.4 & 68.8 \\
4096 & $18.72 \pm 0.54$ & 69.7 & 66.8 \\
16384 & $18.34 \pm 0.36$ & 67.8 & 65.4 \\
\hline
\end{tabular}


that this is the phenomenon which we have observed. Thus, the logarithmic increase in defect density is very likely to be the cause of the logarithmic decrease in the shear elastic constant.

We conclude by comparing our data with those published by Toxvaerd. ${ }^{3}$ He studied the Lennard-Jones system at densities which were about $0.9 \%$ and $1.7 \%$ from melting. For systems of up to 1024 particles he also found a logarithmic decrease as the size of the system increased. His data are shown in Fig. 10. His error bars are somewhat larger than ours. His data are qualitatively the same as ours and refer to states which are also of the order of $1 \%$ from melting. We conclude that the two sets of data are consistent and mutually supportive.

\section{THE PARTICLE DISPLACEMENTS}

The displacements of particles from their lattice sites have been studied in several two-dimensional systems. ${ }^{9,10}$ The main result of these studies is that one can clearly see that the mean-square displacement, $\left\langle u^{2}\right\rangle$, increases logarithmically with the size of the system. The recent study by Toxvaerd $^{3}$ has, however, shown an important new phenomenon. When $\left\langle u^{2}\right\rangle$, measured in units of the near-neighbor distance, reaches a critical value of 0.032 , a diffusion process sets in. The aim of our work was to study this phenomenon in more detail in order to understand the physical processes which are responsible for it, and also to determine the conditions under which it arises. This diffusion process makes it impossible, in some circumstances, to define an equilibrium value for $\left\langle u^{2}\right\rangle$ for a given system. Our results are in good agreement with those of Toxvaerd. We have, however, been able to study larger systems over a wider range of density. This has added to the body of interesting data and we have, we believe, been able to establish the physical mechanism responsible for this diffusion. We believe that the diffusion phenomena will take place at all densities for sufficiently large systems. It is unlikely that it is connected to the melting phenomenon.

\section{A. The size dependence of $\left\langle u^{2}\right\rangle$}

As a preliminary step, we present our data on $\left\langle u^{2}\right\rangle$ for the HD system $3 \%$ from melting, $\rho^{*}=0.942$. For system sizes of 1024,4096 , and 16384 particles $\left\langle u^{2}\right\rangle$ increases logarithmically with the size of the system. Our data (Fig. 11) are in excellent agreement with those of Young and Alder. ${ }^{9}$ At this density there are well-defined values for $\left\langle u^{2}\right\rangle$, and the systems behave as expected. There are large fluctuations in $\left\langle u^{2}\right\rangle$ in the course of one million sweeps in the 16384-particle system, but there is no tendency for $\left\langle u^{2}\right\rangle$ to increase systematically throughout the run. The mean value of $\left\langle u^{2}\right\rangle$ is 0.032 . This is exactly the critical value suggested by Toxvaerd's work. We will return to this point later in this section. The same logarithmic size dependence was found in the $R-12$ system at a density $\rho^{*}=1.06$ which is $5 \%$ above the melting density. These data are also shown in Fig. 11. The very close agreement shown by these two curves again confirms that our method of calibrating the HD and $R-12$ systems is working well. We can thus conclude that at these densi-

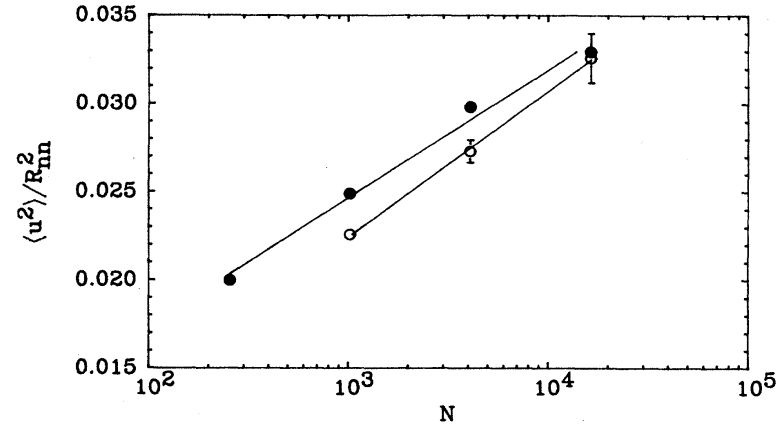

FIG. 11. The size dependence of the mean-square displacement; (O) HD at $\rho^{*}=0.942$; (๑) $R-12$ at $\rho^{*}=1.06$. $R_{n n}$ is the nearest-neighbor distance.

ties and system sizes, where no diffusion occurs, the mean-square displacement increases logarithmically with the size of the system. However, it is quite possible that for even larger systems diffusion will occur.

\section{B. Diffusion at intermediate densities}

We have shown that the HD system at $\rho^{*}=0.923$ matches well with the $R-12$ system at $\rho^{*}=1.04$. This matched pair of densities lies between the higher densities, at which we have just reported, and those much closer to melting. We find that the displacements of the particles are very similar in the two systems at these matched densities. The 1024-particle systems are marginally stable against diffusion. Some diffusion appears to take place, but then for a very large number of passes $\left\langle u^{2}\right\rangle$ remains relatively constant. This is observed in both the HD and $R-12$ systems. For the next largest system the picture changes (Fig. 12). We now have a fairly continuous diffusion process in which $\left\langle u^{2}\right\rangle$ increases steadily in value from about 300000 sweeps onward. In the earlier period of the run, $\left\langle u^{2}\right\rangle$ remains relatively constant. We notice that even in the initial stages of this run $\left\langle u^{2}\right\rangle$ was at least equal to, if not greater, than Toxvaerd's critical value of 0.032 . We have obtained similar, but more limited, data for the $R-12$ system. For a 300000 sweep run the system behaved in a very similar manner.

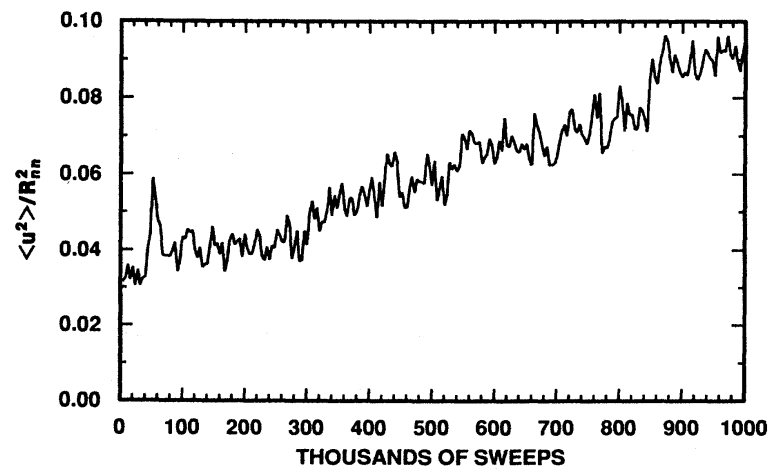

FIG. 12. The control chart for the mean-square displacement for the HD system, 4096 particles, one million sweeps at $\rho^{*}=0.923$. 
The value of $\left\langle u^{2}\right\rangle$ rapidly rose above the critical value of 0.032 , and diffusion set in-although in an irregular fashion. For our largest systems of 16384 particles diffusion set in rapidly, and $\left\langle u^{2}\right\rangle$ reaches much larger values by the end of the run.

\section{Diffusion near the melting point}

We now turn to our data very near melting. For the HD system this is at $\rho^{*}=0.917$, for the $R-12, \rho^{*}=1.02$. These are a pair of matched densities. At these densities diffusion sets in very early in our MC runs. Even the 1024-particle systems show significant diffusion; it is no longer a marginally stable system. In less than 10000 sweeps $\left\langle u^{2}\right\rangle$ reaches the critical value of 0.032 ; diffusion sets in and continues thereafter. Figure 13 shows the control chart for $4096 \mathrm{HD}$ particles and a two million sweep run. The value of $\left\langle u^{2}\right\rangle$ for the $R-12$ system after 300000 sweeps lies on this curve as shown in Fig. 13. This agreement again supports our conjecture that when we have matched densities by comparing the pair distribution functions, then the systems will have very similar properties. In Fig. 13 we also show the control chart for $\left\langle u^{2}\right\rangle$ for two million sweeps for the 16384-particle system. The value of $\left\langle u^{2}\right\rangle$ for the $R-12$ system (not shown in Fig. 13) after 300000 passes in this size system is again very close to the value in the HD system. In this large system diffusion sets in almost immediately and continues in a very smooth fashion. It is interesting to compare the evolution of $\left\langle u^{2}\right\rangle$ as shown by the two curves in this plot. Clearly, the larger system shows a much smoother evolution. However, the smaller system actually shows, on the average, a greater diffusion rate; $\left\langle u^{2}\right\rangle$ reaches a value about $30 \%$ larger in the smaller system. The rather uneven evolution of $\left\langle u^{2}\right\rangle$ in this system is very clearly correlated to the behavior of the pressure and defect density shown in Figs. 2 and 8, respectively.

It is interesting to note that although we see appreciable MC diffusion near melting, it is very much smaller in

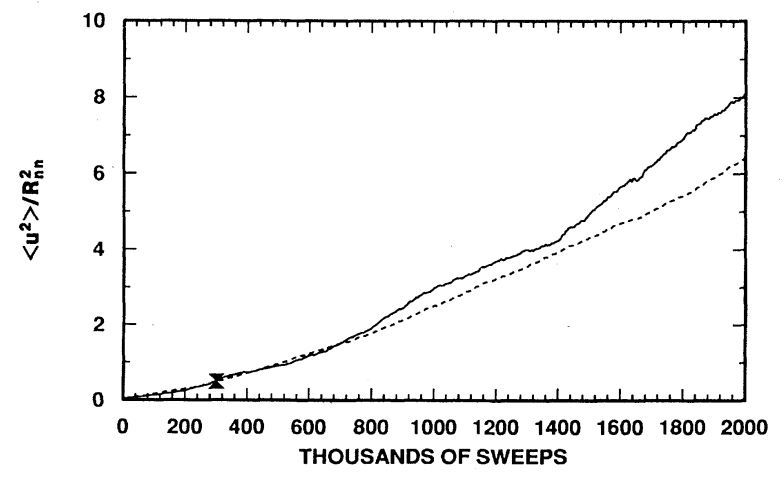

FIG. 13. The control chart for the mean-square displacement for the HD system for two million sweeps at $\rho^{*}=0.917$. System sizes are 4096 particles (solid curve) and 16384 particles (dotted curve). The double triangle symbol shows the value of the mean-square displacement at $\rho^{*}=1.02$ for the $R-12$ system with 4096 particles after 300000 sweeps. magnitude than the MC diffusion in the fluid phase. We have carried out a simulation in the HD fluid at a density of about $0.1 \%$ before freezing. In this very dense fluid the MC diffusion takes place about eight times faster than in the HD solid $0.5 \%$ before melting.

\section{The critical value of $\left\langle u^{2}\right\rangle$}

Our data strongly confirm the suggestion put forward by Toxvaerd-namely, that when $\left\langle u^{2}\right\rangle$ reaches the critical value of 0.032 , diffusion will set in. Toxvaerd was, of course, observing real time diffusion using molecular dynamics; we are observing MC diffusion. We do not believe that this diffusion is directly related to melting. For example, we see substantial diffusion over one million sweeps in the 16384-particle HD system at $\rho^{*}=0.923$ which is $1 \%$ from melting. The control chart for the number of defects for the same run shows no increase in the number of defects. We thus conclude that the diffusion is not leading to an increase in the disorder in the system. In Fig. 1 we showed the control chart for the pressure for the same MC run. As we have already pointed out, there is only a very small drift in the pressure during this long run. Thus, the system is not becoming more disordered, and it is certainly not melting. As a final demonstration of this, we show in Fig. 14 a plot of the positions of approximately 1600 of the particles in the 16 384-particle system after approximately one million sweeps. The particles are still very well ordered even though they have moved on the average by nearly half of the near-neighbor distance. A well-defined triangular lattice is present. In Fig. 15 we show a corresponding plot for the dense fluid phase $\rho^{*}=0.879$. A comparison of Figs. 14 and 15 clearly shows the high degree of triangular order in the solid phase. It is fairly easy to discover what has happened during the $\mathrm{MC}$ run in the solid. Figure 16 shows a plot which reveals which particles have moved from one lattice site to another. Very clearly long-chain or very-long-ring motions have taken place.

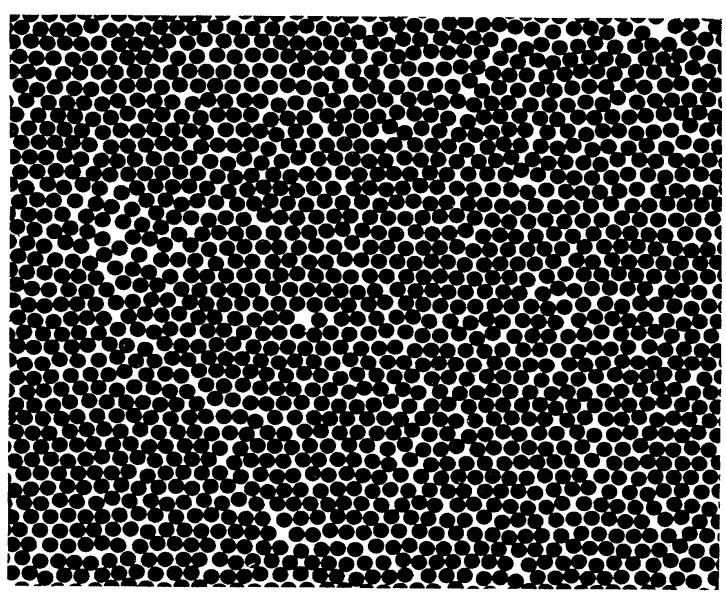

FIG. 14. A snapshot of approximately 1600 HD particles taken from a configuration of 16384 particles. The snapshot is taken after approximately one million sweeps at a density $\rho^{*}=0.923$. 


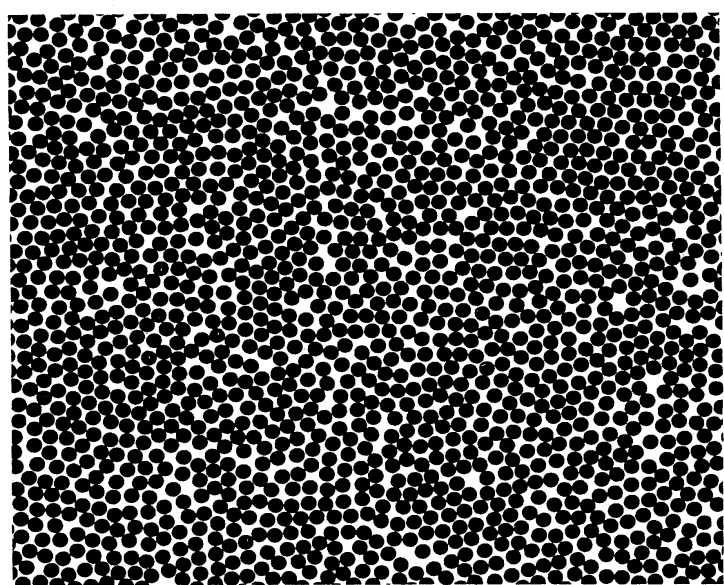

FIG. 15. A snapshot of approximately $1000 \mathrm{HD}$ particles taken from a configuration of 16384 particles after 300000 sweeps at a density $\rho^{*}=0.879$.

In these motions particles move from one lattice site to another without inducing appreciable disorder in the system. This diffusion we see is the consequence of the hopping of particles from lattice site to lattice site, involving large and small rings of particles. It is interesting to note that at this density diffusion is not taking place throughout the system but is confined to localized regions. Very similar motions have been seen in the harddisc system by Alder, Ceperley, and Pollock ${ }^{11}$ and in the two-dimensional Coulomb system by Choquard and Clerouin. ${ }^{12}$ We believe that when $\left\langle u^{2}\right\rangle$ is sufficiently large, gaps open up between the near-neighbor ring of particles, and this allows motion from one site to another. If $\left\langle u^{2}\right\rangle$ is small, then each particle is always surrounded

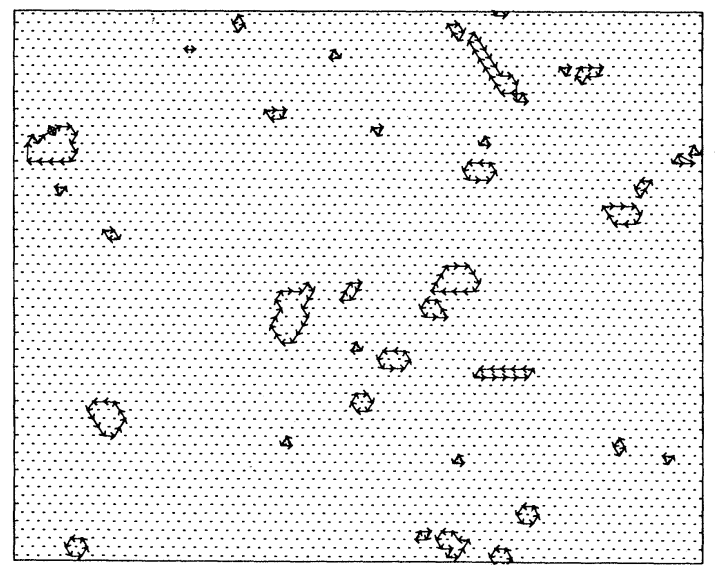

FIG. 16. A plot of the Monte Carlo motions of the particles in a HD system with 4096 particles at $\rho^{*}=0.923$ during a one million sweep run. The arrows show which particles have moved from one site to another. All the other particles have remained within one lattice spacing of their original positions. by a near-neighbor ring which never fluctuates enough to allow the central particle to escape. If this suggestion is correct, then MC diffusion and real time diffusion will set in at any density in the solid provided $\left\langle u^{2}\right\rangle$ is above the critical value. Since we know that before diffusion sets in, $\left\langle u^{2}\right\rangle$ increases logarithmically with system size, we believe that at any density $\left\langle u^{2}\right\rangle$ will always exceed the critical value of 0.032 , provided the system is large enough. We saw in subsection (A) that for 16384 particles and a density of 0.942 the system had just reached, but did not exceed, the critical value of $\left\langle u^{2}\right\rangle$. A crucial test of this argument is to simulate the HD system for 64000 particles at this density $\rho^{*}=0.942$. At this density we predict a value of $\left\langle u^{2}\right\rangle=0.039$ which exceeds the theoretical limit.

We finally note that the critical value of $\left\langle u^{2}\right\rangle$, which is 0.032 , implies that $\left\langle u^{2}\right\rangle^{1 / 2}=0.18$. This is very close to the Lindemann value of $\left\langle u^{2}\right\rangle^{1 / 2}$ in the three-dimensional solid. There are excellent simulation data which show that three dimensional solids melt when $\left\langle u^{2}\right\rangle^{1 / 2}$ reaches a value of approximately 0.17 . The striking similarity of the critical values of $\left\langle u^{2}\right\rangle^{1 / 2}$ in two- and threedimensional systems is worthy of further investigation.

\section{LOCAL AND LONG-RANGE ANGULAR ORDER}

In this section we present a summary of our results on the local and long-range angular order for the hard-disc system. Our success in calibrating the HD and $R-12$ systems leads us to believe that the $R-12$ system will behave in a similar fashion.

\section{A. The local angular order}

The local angular order parameter $\left|\psi_{1}\right|^{2}$ is defined by the equation

$$
\left|\psi_{1}\right|^{2}=\left\langle\left|\frac{1}{n_{l}} \sum_{j}^{n_{l}} e^{6 i \theta_{j}}\right|^{2}\right\rangle .
$$

Here $n_{l}$ is the number of neighbors of the central particle which lie within a circle chosen so that the average number is six. The summation is over these neighbors and $\theta_{j}$ is the angle between the vector joining the $j$ th particle to the central particle and an axis of fixed direction.

We have computed this quantity for the HD system at the densities $\rho^{*}=0.917,0.923$, and 0.942 . The system size varied between 1024 and 16384 particles and our runs were typically of one million sweeps. Our results for this order parameter are very similar to those for the pressure which we reported in Sec. II. This is not surprising as both quantities are local quantities involving near-neighbor configurations only. For the largest system, 16384 particles, at $\rho^{*}=0.923$ there is a small downward drift in the local angular order; this amounts to $0.1 \%$ over one million sweeps. This is almost identical to the behavior of the pressure at this density. At the higher density, $\rho^{*}=0.942$, there is no appreciable drift. Again, this is identical with the behavior of the pressure. At the lower density, $\rho^{*}=0.923$, the system shows a size dependence of about $0.1 \%$ to $0.2 \%$, as does the pressure. There is no size dependence of this magnitude at the 
higher density.

We now turn to our results at the density of 0.917 which is about $0.5 \%$ from the melting density. Both the $16384-$ and 4096-particle systems now show larger drifts. Over one million sweeps the 4096-particle system shows a downward drift in the local-angular order of about 0.5 percent. A similar drift is observed in the 16 384-particle system. We clearly have not been able to establish an equilibrium value for the local order at this density. For the smallest system of 1024 particles we find a relatively stable behavior over one million sweeps. There are, however, large fluctuations about the mean value of the local order. Again we see that the behavior of the local angular order is very similar to that of the pressure.

\section{B. The long-range angular order}

This is defined by the equation

$$
\left|\psi_{N}\right|^{2}=\left\langle\left|\frac{1}{N} \sum_{l}^{N} \frac{1}{n_{l}} \sum_{j}^{n_{l}} e^{6 i \theta_{j}}\right|^{2}\right\rangle \text {. }
$$

The symbols $n_{l}$ and $\theta_{j}$ have the same meaning as before. The summation over $l$ is over all the particles in the system. It is believed ${ }^{13}$ that the quantity will be finite in a two-dimensional solid, and will be of $O(1 / N)$ in the fluid phase.

At the lowest reduced density $\rho^{*}=0.917$, the longrange angular order decreases steadily during the course of a one million sweep run; only for the 1024-particle system do we find a stable value. The decrease is about $2 \%$ for the 4096-particle system. This is much smaller than the increase in the density of defects $(50 \%)$ during the course of the same run. Nevertheless, we believe that the two phenomena are closely related; the increase in the defect density is very likely to be the cause of the decrease in the long-range angular order. When we turn to the two high densities $\rho^{*}=0.923$ and $\rho^{*}=0.942$, we find that the long-range order is now very stable over one million

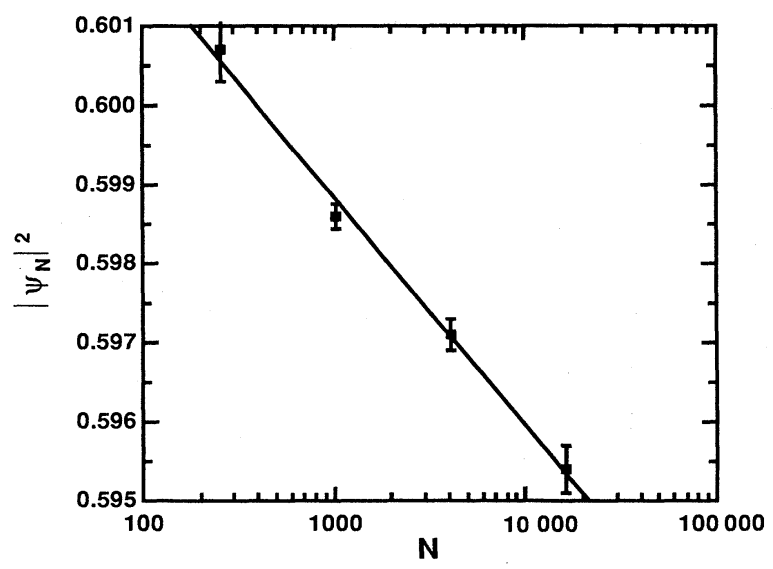

FIG. 17. The long-range angular order parameter, $\left|\psi_{N}\right|^{2}$ as a function of system size $N$. The value of $N$ runs from 256 to 16384 and $\rho^{*}=0.923$.

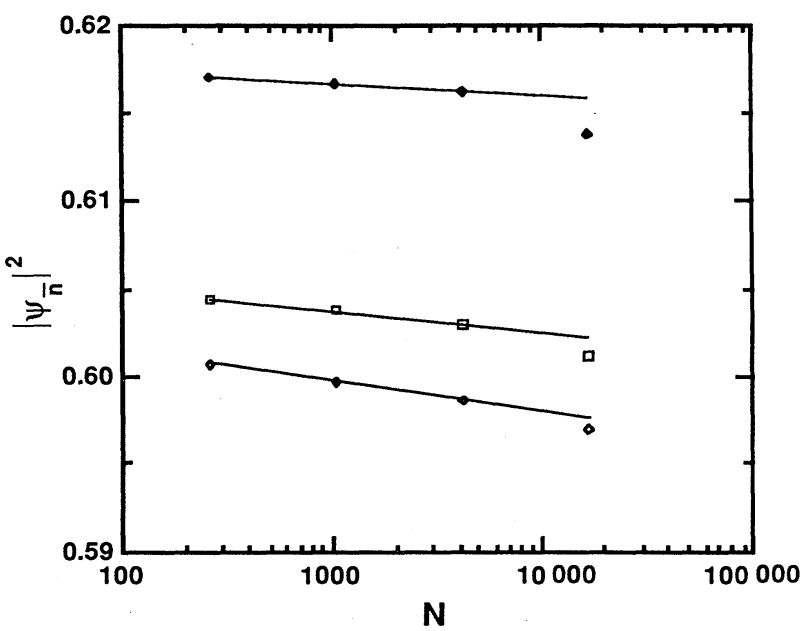

FIG. 18. The intermediate range order parameters $\left|\psi_{\bar{n}}\right|^{2}$. The symbols are as follows: $\bar{n}=256$ is denoted by $\diamond, \bar{n}=64$ by $\square, \bar{n}=16$ by $N$ runs from 256 to 16384 and $\rho^{*}=0.923$.

sweep runs. The downward drifts are now of the order of $0.1 \%$ at most.

We have examined the size dependence of the longrange order at $\rho^{*}=0.923$. It will be recalled that at this density we have found logarithmic size dependences for the density of defects and the shear elastic constant. Our data are shown in Fig. 17. The long-range angular order follows a very accurate logarithmic decay. We can also look at the angular order at intermediate length scales in different size systems. For this purpose we define

$$
\left|\psi_{\bar{n}}\right|^{2}=\left\langle\left|\frac{1}{n} \sum_{l}^{n} \frac{1}{n_{l}} \sum_{j}^{n_{l}} e^{6 i \theta_{j}}\right|^{2}\right\rangle \text {. }
$$

The symbols $n_{l}$ and $\theta_{j}$ have the same meaning as before. Now the summation over $l$ is over all the particles in a cell of a size such that the average number of particles is $\bar{n}$. We denote the actual number of particles in the cell by $n$. This quantity measures the degree of angular order on an intermediate length scale determined by $\bar{n}$ in a system of size $N$. We have computed these order parameters for $\bar{n}=16,64$, and 256 and for system sizes $N=256,1,024$, 4,096, and 16384. The data are shown in Fig. 18. All three order parameters show a logarithmic decay. The decay is less the smaller the value of $\bar{n}$. All the data are for $\rho^{*}=0.923$. For the largest system there is a tendency for the order parameters to drift to smaller values during the one million sweep run. This suggests that this large system is slowly becoming more disordered. These results all point in the same direction. Larger systems are less well ordered than smaller systems.

These logarithmic size dependences are very likely to be closely related to those we found for the density of topological defects. Further investigation with larger systems is likely to lead to a confirmation of these phenomena. In particular, we would like to know whether larger systems at the density of $\rho^{*}=0.942$ will show these phe- 
nomena. If sufficiently large systems do show these logarithmic size dependences at this higher density, then we speculate that they will occur at any density for a sufficiently large system.

\section{DISCUSSION}

We can summarize the results of our simulations rather easily. Our work has been carried out in what appears to be three distinct density regimes: very close to melting ( $0.5 \%$ from melting), about $1 \%$ from melting, and several percent from melting. We will refer to these as the low, intermediate, and high densities. The low-density studies were confined to the hard-disc system. Here, we found that we were unable to establish equilibrium values for the pressure or any other property we studied. The properties drift over long Monte Carlo runs. We conclude that either the melting point of the HD system has been incorrectly located or that the melting of these systems is more complex than a weak first-order transition.

At the intermediate density, about $1 \%$ from melting, we found stable equilibrium values for the properties of both the HD and $R-12$ systems. At the same time the larger systems showed very appreciable Monte Carlo diffusion of the particles. In addition, we found very significant size dependences in the density of defects, the shear elastic constant and the long-range angular order. All showed a logarithmic size dependence which in each case suggested a loss of order or lack of stability of the solid phase. We emphasize that these size dependences were present in what appear to be stable thermodynamic states in which the pressure shows almost no size dependence.

At the higher densities, several percent from melting, neither system shows any significant size dependence up to 16384 particles, and no appreciable Monte Carlo diffusion occurs. However, we now put forward a hypothesis. We suggest that at these higher densities the same size dependences will be found provided the system sizes are large enough so that $\left\langle u^{2}\right\rangle$ exceeds the critical value of 0.032 . As we have pointed out for the HD system at $\rho^{*}=0.942$, the quantity $\left\langle u^{2}\right\rangle$ has almost reached this value for our largest system of 16384 particles. We have established that at this density $\left\langle u^{2}\right\rangle$ increases logarithmically with the size of the system and that our data are in excellent agreement with the results of Young and Adler. If we extrapolate our data to the next largest system of 65536 particles we find a value for $\left\langle u^{2}\right\rangle$ of 0.039 . We thus expect diffusion to set in and a logarithmic size dependence to appear for the other physical properties. To test this hypothesis, we plan to make Monte Carlo runs on systems of 65536 and 262144 particles. We have developed a new MC code for the purpose which will allow us to run four or more FPS M64 array processors in parallel, thus reducing the processing time by a factor of nearly 4 .

\section{ACKNOWLEDGMENTS}

This work was supported by the National Science Foundation under Contract No. DMR-85-16616 through the Material Science Center at Cornell University. The computations were performed using the Cornell National Supercomputer Facility, a resource of the Center for Theory and Simulation in Science and Engineering of Cornell University, which is funded in part by the $\mathrm{Na}$ tional Science Foundation, New York State and the IBM Corporation and members of the Corporate Research Institute.

\section{APPENDIX}

The standard Metropolis Monte Carlo method for generating configurations of a hard-disc system requires exactly two random numbers per attempted displacement of a particle, one for each direction. Thus, for a sweep through a system of $N$ particles, exactly $2 N$ random numbers are needed. Most tests of pseudorandom number generators check for short-interval correlations, but correlations over large intervals, such as $2 N$, can have serious consequences for a simulation of a solid near melting, as we observed.

All of our systems have $N$ equal to a power of four because we use fast Fourier transforms for some of our analysis. The simple linear congruential pseudorandom number generators, like VRAND in the Floating Point Systems APMATH libraries which scale their results modulo a power of two, such as the 28 th or 31 st, have striking correlations at intervals of $2^{n}$, where $n$ is an odd number in the range $11-15$. These can be seen by preparing a map using successive numbers from the sequence as $x$ and $y$ coordinates, and plotting pairs which are separated by $2^{n}$ in the sequence. The locus of points on these maps becomes more severely striped as $n$ increases. These are, in fact, the numbers used to generate the attempted displacements for a single particle in the HD simulation, so as the number of particles included in the simulation increases, the attempted moves of a given particle become less random.

This problem was avoided in two ways. The first way was to use a scheme of employing random numbers which is closer to that used by simulations for soft potentials, namely, that each time an attempted move was rejected (the energy change for the attempted move had increased), a number from the pseudorandom number sequence was thrown away. Thus, the number of random numbers used for a sweep would be $N(2+\alpha)$, where $\alpha$ is the acceptance rate. This effectively eliminated the problem. More recently, the pseudorandom number generator has been changed to DURAND from the IBM ESSL library, which, although it is a linear congruential pseudorandom number generator, does not scale its results by a power of 2 . It has been checked for correlations at intervals of $2^{n}$, and none were found. 
1J. Q. Broughton, G. H. Gilmer, and J. D. Weeks, J. Chem. Phys. 75, 5128 (1981); K. J. Strandburg, Rev. Mod. Phys. 60, 161 (1988).

${ }^{2}$ B. J. Alder and T. E. Wainwright, Phys. Rev. 127, 359 (1962).

${ }^{3}$ S. Toxvaerd, Phys. Rev. Lett. 51, 1971 (1983).

${ }^{4}$ D. Frenkel, and J. P. McTague, Phys. Rev. Lett. 42, 1632 (1979).

${ }^{5}$ H. S. M. Coxeter, Introduction to Geometry, 2nd ed. (Wiley, New York, 1961).

${ }^{6}$ G. L. Dirichlet, J. Reine Angew. Math. 40, 209 (1850).

${ }^{7}$ D. R. Squire, A. C. Holt, and W. G. Hoover, Physica 42, 388
(1968).

${ }^{8}$ B. I. Halperin and D. R. Nelson, Phys. Rev. B 19, 2457 (1979). ${ }^{9}$ D. A. Young and B. J. Alder, J. Chem. Phys. 60, 1254 (1974).

${ }^{10}$ R. C. Gann, S. Chakravarty, and G. V. Chester, Phys. Rev. B 20, 326 (1979).

${ }^{11}$ B. J. Alder, D. M. Ceperley, and E. L. Pollock, Int. J. Quantum Chem. Symp. 16, 49 (1982).

${ }^{12} \mathrm{Ph}$. Choquard and J. Clerouin, Phys. Rev. Lett. 50, 2086 (1983).

${ }^{13}$ N. D. Mermin, Phys. Rev. 158, 383 (1967). 